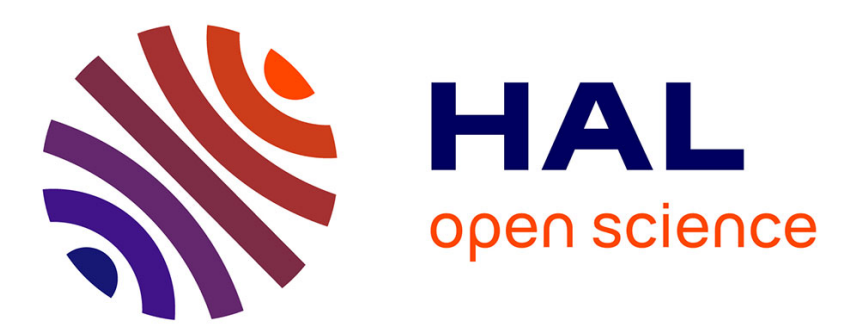

\title{
Effect of phase space folding on the accuracy of the Berk-Breizman cubic equation
}

D. Sanz-Orozco, H.L. Berk, Matteo Faganello, M. Idouakass, Gang Wang

\section{To cite this version:}

D. Sanz-Orozco, H.L. Berk, Matteo Faganello, M. Idouakass, Gang Wang. Effect of phase space folding on the accuracy of the Berk-Breizman cubic equation. Nuclear Fusion, 2018, 58 (8), pp.082012. 10.1088/1741-4326/aab84a . hal-03101829

\section{HAL Id: hal-03101829 \\ https://hal.science/hal-03101829}

Submitted on 7 Jan 2021

HAL is a multi-disciplinary open access archive for the deposit and dissemination of scientific research documents, whether they are published or not. The documents may come from teaching and research institutions in France or abroad, or from public or private research centers.
L'archive ouverte pluridisciplinaire HAL, est destinée au dépôt et à la diffusion de documents scientifiques de niveau recherche, publiés ou non, émanant des établissements d'enseignement et de recherche français ou étrangers, des laboratoires publics ou privés. 


\title{
Effect of phase space folding on the accuracy of the Berk-Breizman cubic equation
}

\author{
D. Sanz-Orozco ${ }^{1} \oplus$, H.L. Berk ${ }^{2}$, M. Faganello ${ }^{3}$, M. Idouakass ${ }^{4}$ and G. Wang ${ }^{2}$ \\ ${ }^{1}$ University of Tampa, Tampa, FL 33606, United States of America ${ }^{\mathrm{a}}$ \\ 2 Institute for Fusion Studies, University of Texas, Austin, TX 78712, United States of America \\ 3 Aix-Marseille Université, CNRS, PIIM UMR 7345, 13397 Marseille, France \\ ${ }^{4}$ Institut Jean-Lamour, UMR 7198 CNRS, Université de Lorraine, 54506 Vandoeuvres-lès-Nancy, France
}

Received 3 December 2017, revised 11 March 2018

Accepted for publication 21 March 2018

Published

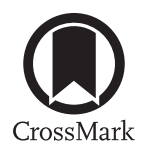

\begin{abstract}
We assess the quantitative accuracy of the Berk-Breizman (BB) cubic wave equation that has been previously derived to describe the initial nonlinear behavior of kinetic instabilities (Berk et al 1996 Phys. Rev. Lett. 76 1256-9; Breizman et al 1997 Phys. Plasmas 4 1559-68; Berk et al 1997 Plasma Phys. Rep. 23 778-88). The fifth-order terms in the wave equation are calculated for the first time, which allow us to quantify this accuracy arbitrarily close to marginal stability. We find a relation between the breakdown of the quantitative accuracy of the cubic equation, occurring well below the instability's saturation level, and the recently observed phase space folding effect (Idouakass 2016 PhD Thesis Aix-Marseille University). This effect emerges when the resonant particle distribution function $f(\varphi, \Omega, t)(\Omega$ is a momentum-like variable, $\varphi$ is its conjugate variable, and $t$ is the time) evolves from a (locally) single-valued function of $\varphi$, at fixed $\Omega$, to a triple-valued one (i.e. there emerges a point in phase space where $\partial f / \partial \Omega=0)$.
\end{abstract}

Keywords: nonlinear waves, weak nonlinearity, energetic particle modes, phase space dynamics

AQ3 (Some figures may appear in colour only in the online journal)

\section{Introduction}

The evolution of nonlinear waves that are destabilized by energetic particles is a widely studied problem in plasma physics. Significant insight into the problem has been gained by studying the near-threshold regime [1-3], where the particle destabilization is nearly balanced by background dissipation. This setting often develops in real-world fusion devices, because the population of energetic particles (namely $\alpha$ particles) builds up until it overcomes background dissipation. Indeed, the near-threshold theory has been used to interpret several fusion plasma experiments [5-8], in which toroidal Alfvén eigenmodes (TAEs) are destabilized by energetic particles. In general, these energetic-particle-driven TAE

${ }^{\text {a }}$ Work carried out in part while at University of Texas at Austin. instabilities require a three-dimensional description; however, the comparatively-simple near-threshold model is still applicable because near a resonant surface it is possible to reduce the problem to a one-dimensional one through a canonical transformation in an action-angle formalism [2, 3]. In this article, we study the limit of validity of the near-threshold theory. Before embarking on this detailed study, a brief introduction to the theory is given in this section.

In the original derivation of the theory, Berk et al [1] considered the classic bump-on-tail problem, where an electrostatic wave resonates with an inverted fast-particle population. The authors restricted their study to the case of isolated resonances close to marginal stability, a restriction that permits a perturbative expansion from which the authors derived a wave amplitude equation. More specifically, the expansion results in a series of increasingly nonlinear terms in the wave

\begin{tabular}{|lllll}
\hline JNL:NF & PIPS: AAB84A & TYPE: SPE & TS: NEWGEN & DATE:28/3/18
\end{tabular}


equation. As long as the mode amplitude is sufficiently small, a truncation of the series is justified where only the lowestorder nonlinear term, which is cubic in the mode amplitude, is kept. The resulting cubic wave equation, also commonly known as the Berk-Breizman (BB) equation, thus captures the onset of nonlinear effects (i.e. the equation constitutes a theory of weak nonlinearity).

Through the cubic equation, Berk et al found four main regimes of evolution for the weakly-nonlinear wave, with the regime depending upon the relative strength of two competing effects: (1) the tendency of the electrostatic wave to flatten the fast-particle distribution at the resonance location, and (2) the tendency of relaxation processes (i.e. collisions) to restore the inverted distribution. The most strongly nonlinear regime, referred to as the 'explosive' regime, is entered if relaxation processes are relatively weak. As opposed to the other three regimes, the explosive evolution eventually takes the theory outside of its domain of validity as the wave amplitude grows without bound, becoming singular in a finite time.

The BB cubic equation displays temporal nonlocality, and it is commonly written in terms of dimensionless variables as

$$
\begin{aligned}
\frac{\mathrm{d} A}{\mathrm{~d} \tau}= & A(\tau)-\frac{1}{2} \int_{0}^{\tau / 2} \mathrm{~d} \tau_{1} \tau_{1}^{2} A\left(\tau-\tau_{1}\right) \\
& \times \int_{0}^{\tau-2 \tau_{1}} \mathrm{~d} \tau_{2} A\left(\tau-\tau_{1}-\tau_{2}\right) A^{*}\left(\tau-2 \tau_{1}-\tau_{2}\right),
\end{aligned}
$$

where $A=\omega_{b}^{2} \gamma_{\mathrm{L}}^{1 / 2} / \gamma^{5 / 2}$ is a normalized mode amplitude, with $\left|\omega_{b}\right|$ being the bounce frequency of the deeply wave-trapped resonant particles (e.g. in one-dimensional electrostatic sinusoidal oscillations, $\left|\omega_{b}\right|^{2}=q k|E| / m$, where $q, m, k$, and $|E|$ are respectively: particle charge and mass, wave number and electric field wave amplitude). The time has been normalized to units of the linear growth rate $\gamma \equiv \gamma_{\mathrm{L}}-\gamma_{d}$, where $\gamma_{\mathrm{L}}$ is the linear growth rate in the absence of dissipation, and $\gamma_{d}$ is the background dissipation rate. Closeness to marginal stability means that $\gamma \ll \gamma_{\mathrm{L}} \sim \gamma_{d}$.

The modes treated in [1] exist independently of the kinetic contribution from the fast-particle population. These modes are thus described as being perturbative. The near-threshold theory has been generalized to include nonperturbative modes $[2,3]$, oscillations whose very existence requires the fastparticle contribution. This generalization leaves equation (1) unchanged with the exception of a constant phase factor that multiplies the cubic term. In this work, we limit ourselves to the case of perturbative modes, and while we expect our results to carry over to the nonperturbative case, the details of this extension still need to be ascertained. In equation (1) we are also neglecting collisional effects. This approximation can be justified in the strongly-nonlinear case that we will study $[1-3,9]$, although dynamical friction could have non-negligible effects on the amplitude evolution $[10,11]$.

In the $\mathrm{PhD}$ thesis by Idouakass [4] it is noted that, well before the explosive regime of the cubic equation, folding of the isocontours of the resonant particle distribution function $f$ arises in phase space. Here folding means that $f(\varphi, \Omega, t)$ evolves from a (locally) single-valued function of $\varphi$, at fixed

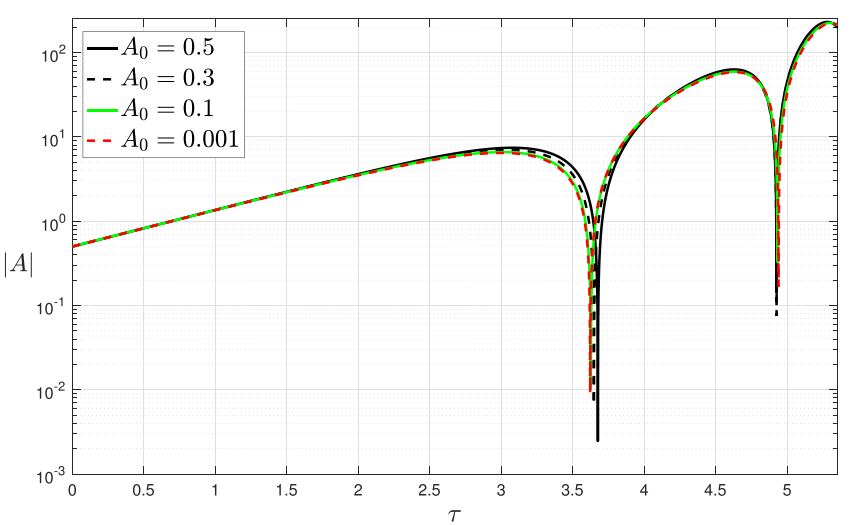

Figure 1. Effect of initial amplitude, $A_{0}$, on the evolution of $|A|$ as given by equation (1). The run with $A_{0}=0.5$ was initiated with this amplitude at $\tau=0$. The other four runs have been shifted in $\tau$ to match the $A_{0}=0.5$ run at $\tau=0$.

$\Omega$, to a triple-valued one. That is, a point where $\partial f / \partial \Omega=0$ emerges in phase space. The folding effect arises only when there is a breakdown of the original assumptions for obtaining the cubic equation which, as we shall discuss, occurs when the $\Omega$-derivative of the perturbed distribution function $f-F$ reaches the value of that of the equilibrium function $F$. This raises the question of whether the cubic equation can provide valid predictions for calculating the mode amplitude even though folding takes place. If the equation remains accurate, as it will be shown to be the case, then the assumptions of the theory were too restrictive. At the same time, we will show that folding eventually leads to a loss of accuracy of the cubic equation well below the amplitude level expected in the original theory. Indeed, it was expected that the predictions of the cubic equation would be quantitatively valid as long as $\left|\omega_{b}(t)\right| \ll \gamma_{\mathrm{L}}$, whereas we will find that the accuracy is lost at far smaller levels of $\left|\omega_{b}\right|$, when it has increased by a factor $\sim 5-10$ from the time when folding arises.

The nonlinear term in equation (1) becomes important when $|A| \gtrsim 1$, and the mode eventually enters the explosive regime, where the mode amplitude scales as $\left(\tau_{0}-\tau\right)^{-5 / 2}$ [1], with $\tau_{0}$ being a blow-up time that only depends upon the initial condition $A(\tau=0)$. When the evolution is deep into the explosive regime (roughly after a few 'bounces' of $|A|$ ), the initial amplitude becomes unimportant, as the amplitude response asymptotes to an attractor [9]. If the amplitude is not yet in this asymptotic regime, the behavior can still be considered universal if the initial amplitude is sufficiently small. By 'universal' it is meant that the plot of $A$ as a function of $\tau$ is independent of any parameter, up to an overall shift in $\tau$ (i.e. the response due to a small initial amplitude will begin to replicate the evolution of a larger initial amplitude after it "catches on' to the larger initial condition). This is shown in figure 1 where four simulation runs with different initial amplitudes are shifted in $\tau$ such that they match at $\tau=0$. The ensuing evolution for $\tau>0$ quickly converges with decreasing initial amplitude $A_{0}$; at the first minimum location, the difference between runs is unnoticeable for $A_{0} \lesssim 0.1$. The step size in these runs is $\Delta \tau=0.002$. 
The unbounded growth of the explosive regime implies that, eventually, the equation is taken outside its domain of validity, and only a full-Vlasov simulation will accurately describe the subsequent long-term evolution. Prior to this breakdown, there is always a finite time window where the nonlinear behavior predicted by the cubic equation has physical validity, as long as the parameter $\gamma / \gamma_{\mathrm{L}}$ is sufficiently small. Decreasing the value of this parameter will increase the width of the time window. In other words, the actual mode amplitude (given by full-Vlasov simulations) is expected to replicate the universal cubic solution to an increasingly higher extent as $\gamma / \gamma_{\mathrm{L}}$ is made smaller.

In the present work, full-Vlasov simulations have been performed by improving upon a previously-existing Vlasov code [12]. The code solves for the particle distribution function by discretizing phase space. The improved version uses a Runge-Kutta-Fehlberg method [13] to make the time-step size adaptive. The simulated equations in this fully nonlinear scheme are

$$
\frac{\mathrm{d} \omega_{b}^{2}}{\mathrm{~d} t}=-\frac{\gamma_{\mathrm{L}}}{\pi^{2}} \int \mathrm{d} \varphi \int \mathrm{d} \Omega \tilde{f}(\varphi, \Omega, t) \mathrm{e}^{-\mathrm{i} \varphi}-\gamma_{d} \omega_{b}^{2}
$$

and

$$
\frac{\partial \tilde{f}}{\partial t}+\Omega \frac{\partial \tilde{f}}{\partial \varphi}+\operatorname{Re}\left(\omega_{b}^{2} \mathrm{e}^{\mathrm{i} \varphi}\right) \frac{\partial \tilde{f}}{\partial \Omega}=0
$$

where $\varphi$ is a spatial coordinate in the reference frame of the wave, with associated wave-frame velocity $\Omega \equiv \dot{\varphi}(\Omega=0$ corresponds to resonance), $\omega_{b}^{2}$ is proportional to the wave amplitude (see discussion following equation (1)), and $\tilde{f}$ is a normalized particle distribution function. The term $-\gamma_{d} \omega_{b}^{2}$ models the damping of the wave due to the bulk of the plasma. The initial conditions implemented in our full-Vlasov simulations are a small amplitude $\omega_{b}^{2}(t=0)$ and an equilibrium distribution $\tilde{f}(\varphi, \Omega, t=0)=\Omega$ (i.e. initially $\partial \tilde{f} / \partial \Omega=1$ ).

The parameters $\gamma_{\mathrm{L}}$ and $\gamma_{d}$ in equation (2) are chosen depending upon the desired closeness to marginal stability. The actual linear growth rate of the simulated mode does not turn out to be exactly $\gamma_{\mathrm{L}}-\gamma_{d}$ due to two main reasons: there is an intrinsic error due to discretization, and there is a truncation in the size of the velocity domain. We obtain the true linear growth rate by directly measuring it from the simulated amplitude evolution, and from this measurement we determine the value of $\gamma / \gamma_{\mathrm{L}}$. The other parameters in the code are the size of the simulation box in phase space, the real-space and velocity-space resolutions of the box, and the time step.

Figure 2 shows full-Vlasov simulations of the mode amplitude for two different values of $\gamma / \gamma_{\mathrm{L}}$ (red and blue curves), together with the evolution given by the cubic equation (dotted curve). For the simulation run with $\gamma / \gamma_{\mathrm{L}} \approx 0.0094$, we have used a phase space box with 18000 grid points in the velocity direction and 60 points along the spatial direction, together covering the domain $\Omega / \gamma \in(-267,267), \varphi \in[-\pi, \pi)$. The time step for this run is such that $\gamma \Delta t \equiv \Delta \tau \approx 9.36 \times 10^{-4}$. For the run with $\gamma / \gamma_{\mathrm{L}} \approx 0.0460$, we used 7000 points in velocity and 60 in real space, covering the domain $\Omega / \gamma \in(-560,560), \varphi \in[-\pi, \pi)$.

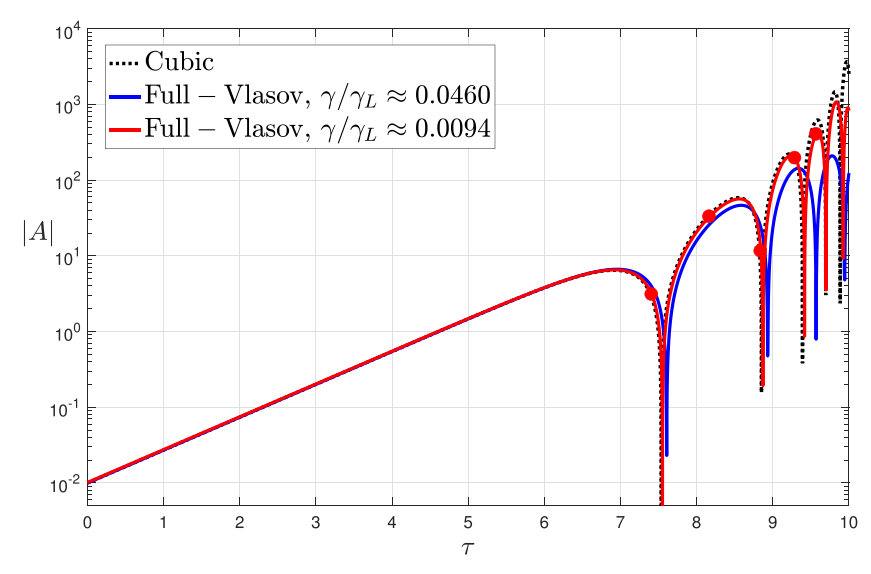

Figure 2. The resemblance between full-Vlasov simulations and the universal cubic response is improved for decreasing values of $\gamma / \gamma_{\mathrm{L}}$. Snapshots of the phase space dynamics are shown in figure 5 for the case of $\gamma / \gamma_{\mathrm{L}} \approx 0.0094$ at the moments indicated in this picture by red dots.

The time step is such that $\gamma \Delta t \equiv \Delta \tau \approx 4.82 \times 10^{-3}$. We see in figure 2 that, indeed, the run with the smaller value of $\gamma / \gamma_{\mathrm{L}}$ is closer to the response of the cubic equation. We will make use of these full-Vlasov simulations throughout this article.

\section{Folding of the distribution function}

In this section we first show that the assumptions in the original derivation [1] of the cubic equation are too stringent. The assumptions that we are discussing are those related to the distribution function components, namely that

$$
\left|F^{\prime}\right| \gg\left|f_{1}^{\prime(1)}\right| \gg\left|f_{0}^{\prime(2)}\right|,\left|f_{2}^{\prime(2)}\right|,
$$

where $F \equiv f(t=0)$ is an equilibrium distribution that is taken to have a constant velocity gradient over all of space, and $f_{n}^{(k)}$ is the $n$th Fourier component of the perturbed distribution $f-F$, with the superscript $(k)$ indicating the order in the mode amplitude. Prime notation indicates a partial derivative with respect to the velocity $\Omega$. Thus $F^{\prime}$ is a constant. We will show that the breaking of this hierarchy, within the near-threshold theory, allows for a 'folding' of the distribution function's contour lines in phase space (the meaning of this statement will be made precise shortly). We note at this point that the distribution $\tilde{f}$ in equations (2) and (3) is a normalized version of the full distribution $f$ that we now treat, with the normalization factor being $F^{\prime}$; thus $\tilde{f}=f / F^{\prime}$.

The derivation in [1] states the assumption in equation (4) without the derivatives with respect to $\Omega$. However, the assumption involving the derivatives appears to be the more appropriate condition given how the Fourier components enter into the equations, as we will see below. Here it will be shown that neither of these assumptions are necessarily satisfied while the cubic equation is accurate. Let us now review the iterative procedure that results from assuming equation (4).

In the Vlasov equation, the $n$th Fourier component of the distribution function is coupled to its neighboring components $f_{n \pm 1}$ via 


$$
\frac{\partial f_{n}}{\partial t}+\mathrm{i} n \Omega f_{n}+\frac{1}{2}\left(\omega_{b}^{2} f_{n-1}^{\prime}+\omega_{b}^{2 *} f_{n+1}^{\prime}\right)=0 .
$$

Solving for $f_{n}$ we find

$$
f_{n}=-\frac{1}{2} \int_{0}^{t} \mathrm{~d} t_{1}\left(\omega_{b}^{2} f_{n-1}^{\prime}+\omega_{b}^{2 *} f_{n+1}^{\prime}\right) \exp \left[-\mathrm{i} n \Omega\left(t-t_{1}\right)\right] .
$$

Note that because $f$ is real, $f_{-n}=f_{n}^{*}$. A small caveat to equation (6) is that in the $n= \pm 1$ case, the equilibrium distribution function $F$ must also be included:

$$
f_{1}=-\frac{1}{2} \int_{0}^{t} \mathrm{~d} t_{1}\left[\omega_{b}^{2}\left(F^{\prime}+f_{0}^{\prime}\right)+\omega_{b}^{2 *} f_{2}^{\prime}\right] \exp \left[-\mathrm{i} \Omega\left(t-t_{1}\right)\right] .
$$

Following [1-3], it is assumed that equation (4) is valid, whereby $f_{0}^{\prime}$ and $f_{2}^{\prime}$ are ignored in equation (7) in order to obtain the lowest order term in the distribution function perturbation, which is

$$
f_{1}^{(1)}=-\frac{1}{2} \int_{0}^{t} \mathrm{~d} t_{1} \omega_{b}^{2}\left(t_{1}\right) F^{\prime} \exp \left[-\mathrm{i} \Omega\left(t-t_{1}\right)\right] .
$$

We then use this expression to find $f_{0}^{(2)}$ and $f_{2}^{(2)}$ (through equation (6) with $n=0$ and $n=2$, respectively), which are thus quadratic in the mode amplitude. In finding $f_{2}^{(2)}$, we ignore $f_{3}^{\prime}$, assuming it is much smaller than $f_{1}^{\prime}$. As a result of this iterative procedure, the $k$ th order term scales as $f_{n}^{(k)} \sim\left(F^{\prime} / t\right)\left(\omega_{b} t\right)^{2 k}$, with $t$ being a relevant time scale.

Let us now test the assumption that $\left|F^{\prime}\right| \gg\left|f_{0}^{\prime(2)}\right|$, which is apparently the justification for the iterative procedure described above. We change the notation to the dimensionless $A$ and $\tau$, and we find that

$$
\begin{aligned}
\frac{\gamma_{\mathrm{L}} f_{0}^{\prime(2)}}{\gamma F^{\prime}}=-\frac{1}{4} \int_{0}^{\tau} \mathrm{d} \tau_{1} A\left(t_{1}\right) \int_{0}^{\tau_{1}} \mathrm{~d} \tau_{2} A^{*}\left(\tau_{2}\right)\left(\tau_{1}-\tau_{2}\right)^{2} \\
\times \exp \left[\mathrm{i} \frac{\Omega}{\gamma}\left(\tau_{1}-\tau_{2}\right)\right]+\text { c.c. }
\end{aligned}
$$

Recall that $A$ is a universal function of $\tau$ as described in section 1 . Therefore, the quantity $\gamma_{\mathrm{L}} f_{0}^{\prime(2)} /\left(\gamma F^{\prime}\right)$ above only depends upon the normalized time $\tau$ and the normalized velocity $\Omega / \gamma$. Equation (9) can be used to numerically search for the time when $f_{0}^{\prime(2)} / F^{\prime}=1$ is first reached, which would violate the assumed ordering used in the derivation of the cubic equation. As an example, consider the case $\gamma / \gamma_{\mathrm{L}} \approx 0.0094$ whose amplitude evolution was plotted in figure 2 (red curve). For this example, $\gamma_{\mathrm{L}} f_{0}^{\prime(2)} /\left(\gamma F^{\prime}\right) \approx 106.8$ when the ordering breaks. Using equation (9), we find numerically that this value is first reached at a time $\tau \approx 8.11$ (the ordering first breaks at $\Omega=0$, as one would expect). Notice that at that time the cubic solution is still a reasonable approximation, as can be seen in figure 2 . The accuracy of the approximation will be quantified in section 3 .

For the same $\gamma / \gamma_{\mathrm{L}} \approx 0.0094$ case, let us now study the evolution of the Fourier components of the perturbed distribution function. Up to third order in the mode amplitude,

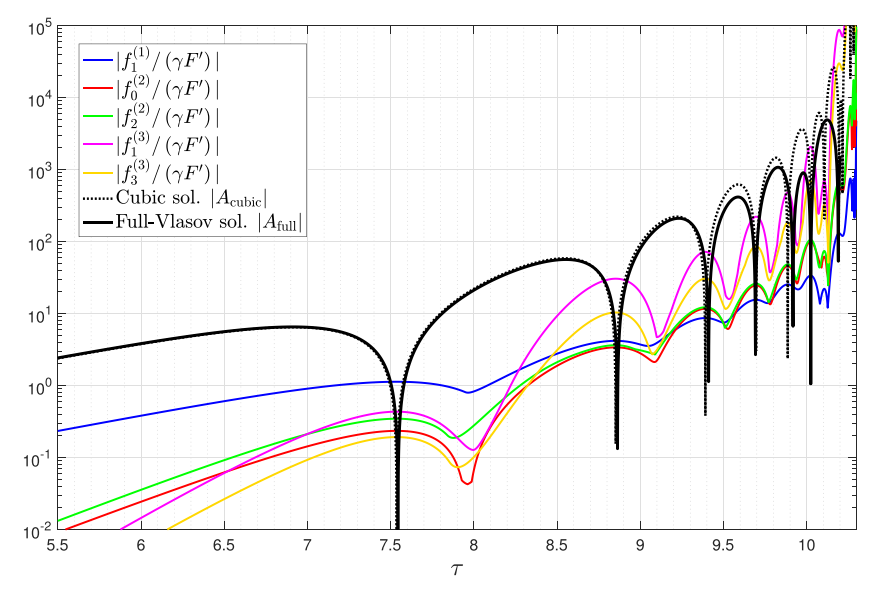

(a)

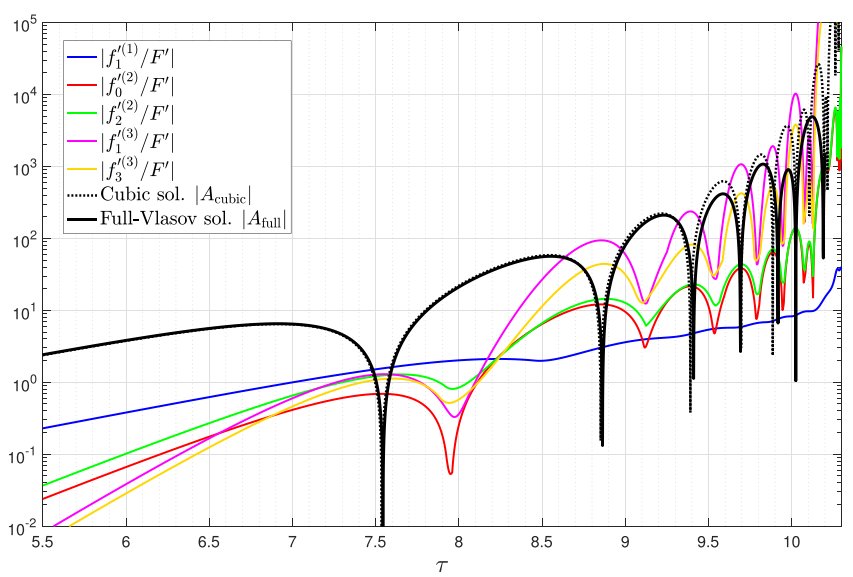

(b)

Figure 3. Evolution of $(a)$ the distribution function's Fourier components and $(b)$ their velocity gradients, both for $\gamma / \gamma_{\mathrm{L}} \approx 0.0094$. The mode amplitude is shown for reference. The simulation begins at $\tau=0$ with an initial amplitude $A_{0}=0.01$; here, only the nonlinear stage is shown, with the first peak of $|A|$ being the one at $\tau \approx 6.9$.

these terms are $f_{1}^{(1)}, f_{0}^{(2)}, f_{2}^{(2)}, f_{1}^{(3)}$ and $f_{3}^{(3)}$. The explicit expressions for these terms are given in appendix A. Note that these components are functions of both $\Omega / \gamma$ and $\tau$. However, in what follows, we only want a measure of the size of the Fourier components, so at every time $\tau$ we find the maximum value of each component (i.e. we evaluate the components over a suitable range of $\Omega / \gamma$ values and select the largest value of the component), and it is this maximum value which we plot in panel $(a)$ of figure 3 . In this figure, the distribution function has been normalized to units of $\gamma F^{\prime}$. For reference, both the cubic and full-Vlasov solutions are shown. Notice that the hierarchy of the Fourier components breaks down at some point before $\tau \approx 8.3$, but the cubic equation has very good accuracy at that time.

A similar finding occurs when we now look at the hierarchy of the derivatives, $f_{1}^{\prime(1)}, f_{0}^{\prime(2)}, f_{2}^{\prime(2)}, f_{1}^{\prime(3)}$ and $f_{3}^{\prime(3)}$. Their evolution in units of $F^{\prime}$ is shown in panel $(b)$ of figure 3 , where again, we evaluate the derivatives at the $\Omega / \gamma$-value where they 


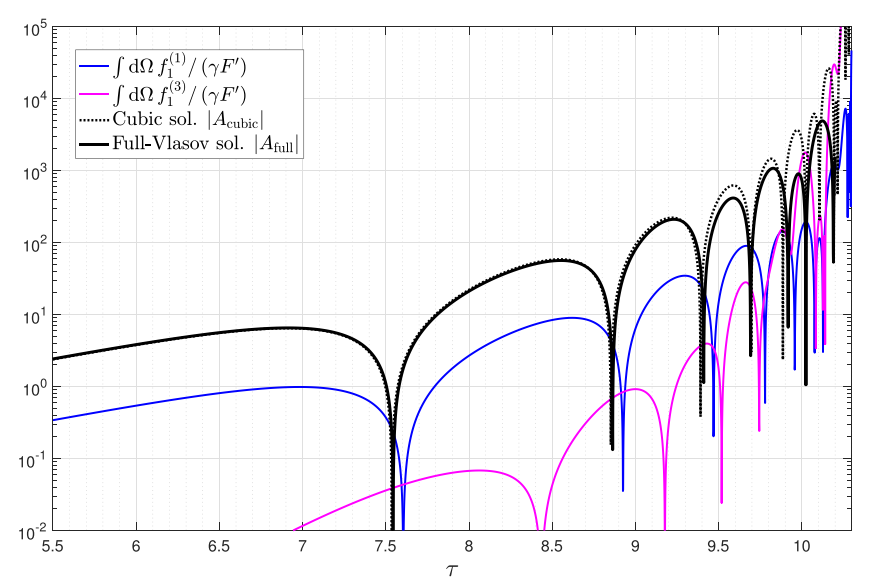

Figure 4. Evolution of the linear and cubic parts of the $n=1$ Fourier component of the current density (up to a constant). Cubic and full-Vlasov solutions shown for reference.

are largest. The hierarchy is broken well within the window where the cubic equation is valid.

We thus have a situation where the assumptions that lead to the cubic wave equation are inaccurate, but yet the prediction of the equation is accurate. To resolve this situation, notice that the kinetic contribution to the wave equation is not proportional to the distribution function components themselves or to their derivatives, but actually depends upon the currents that transfer power to the wave. In other words, the distribution function enters through the ' $\mathbf{J} \cdot \mathbf{E}$ ' term in the wave equation. Specifically, it is only the $n=1$ Fourier component that contributes to this power transfer. The current that contributes to the wave equation is

$$
J_{1} \propto \int_{-\infty}^{\infty} \mathrm{d} \Omega f_{1},
$$

where $f_{1}$ is composed of terms of differing order in the mode amplitude. The leading term is $f_{1}^{(1)}$ (equation (8)), and next in order is $f_{1}^{(3)}$, the latter leading to the cubic term in the wave equation. In figure 4 , we see the evolution of $\int_{-\infty}^{\infty} \mathrm{d} \Omega f_{1}^{(1)}$ and $\int_{-\infty}^{\infty} \mathrm{d} \Omega f_{1}^{(3)}$.

Notice that the separation between $\int_{-\infty}^{\infty} \mathrm{d} \Omega f_{1}^{(1)}$ and $\int_{-\infty}^{\infty} \mathrm{d} \Omega f_{1}^{(3)}$ in figure 4 lasts for a considerably longer time when compared to that between $f_{1}^{(1)}$ and $f_{1}^{(3)}$, or between $f_{1}^{\prime(1)}$ and $f_{1}^{\prime(3)}$ (in figure 3). The hierarchy in the currents is considerably more robust than that of the distribution function components themselves. Thus, we conclude that while the assumed hierarchy of the Fourier components in equation (4) may be broken, the cubic equation may remain valid because it is the integral of the components in velocity-space which enters the wave equation. Notice that the eventual breaking of the hierarchy $J_{1}^{(1)} \gg J_{1}^{(3)}$ is much more indicative of the breakdown of the cubic equation, as can be appreciated in figure 4.

As discussed in section 1, a folding of the distribution function isocontours arises when $f^{\prime}=0$ at some point in phase space. This implies that folding arises at a point where $\left|f^{\prime}-F^{\prime}\right| \sim\left|F^{\prime}\right|$, where $f^{\prime}-F^{\prime}$ is the perturbed distribution function, depending upon the perturbed components $\left|f_{1}^{\prime(1)}\right|$, $\left|f_{0}^{\prime(2)}\right|,\left|f_{2}^{\prime(2)}\right|$, etc. Therefore, one of the apparent consequences of imposing the ordering $\left|F^{\prime}\right| \gg\left|f_{1}^{\prime(1)}\right| \gg\left|f_{0}^{\prime(2)}\right|,\left|f_{2}^{\prime(2)}\right|$ is the implication that a folding is not possible within the validity of the cubic equation. We will show that this implication is not true, and in section 3 we will see that there is a relation between the amplitude level where folding happens, and that at which the cubic equation ultimately loses accuracy.

Before embarking on a general discussion, consider once again the sample case where $\gamma / \gamma_{\mathrm{L}} \approx 0.0094$. Figure 5 shows contour plots of the distribution function at the five instances indicated in figure 2 by red dots. The phase space portrait in panel $(a)$ of figure 5 shows the first instance of a folding event (at $\varphi=0$ ). Note that the mode amplitude is well described by the cubic solution at that time (figure 2).

Let us now predict, for arbitrary $\gamma / \gamma_{\mathrm{L}}$, the first instance of folding. Note that initially $f^{\prime}(\varphi, \Omega, t=0)=F^{\prime}$ for all $\varphi$ and all $\Omega$. As time advances, the perturbations in $f^{\prime}$ grow and folding occurs when, at some point in phase space, the perturbative terms add up to $-F^{\prime}$. By expanding the distribution function in a Fourier series, the folding condition is written as

$$
0=F^{\prime}+f_{0}^{\prime}+\sum_{n=1}^{\infty}\left[f_{n}^{\prime} \exp (\text { in } \varphi)+\text { c.c. }\right] \text {. }
$$

We may write the previous expression indicating the order of the terms:

$$
\begin{aligned}
0=F^{\prime} & +2 \operatorname{Re}\left[f_{1}^{\prime(1)} \exp (\mathrm{i} \varphi)\right]+f_{0}^{\prime(2)} \\
& +2 \operatorname{Re}\left[f_{2}^{\prime(2)} \exp (2 \mathrm{i} \varphi)\right]+\mathcal{O}\left(\omega_{b}^{6} t^{6}\right) .
\end{aligned}
$$

We will estimate the folding level by only keeping the zeroth- and first-order terms above; later we shall comment on the validity of this truncation. Making use of the explicit expression for $f_{1}^{(1)}$ (equation (8)), the folding condition reduces to

$$
\begin{aligned}
0= & +\operatorname{Re}\left[\mathrm{i} \exp (\mathrm{i} \varphi) \int_{0}^{t} \mathrm{~d} t_{1} \omega_{b}^{2}\left(t_{1}\right)\left(t-t_{1}\right)\right. \\
& \left.\times \exp \left[-\mathrm{i} \Omega\left(t-t_{1}\right)\right]\right] \\
= & 1+\left(\frac{\gamma}{\gamma_{\mathrm{L}}}\right)^{1 / 2} \operatorname{Re}\left[\mathrm{i} \exp (\mathrm{i} \varphi) \int_{0}^{\tau} \mathrm{d} \tau_{1} A\left(\tau_{1}\right)\left(\tau-\tau_{1}\right)\right. \\
& \left.\times \exp \left[-\mathrm{i} \frac{\Omega}{\gamma}\left(\tau-\tau_{1}\right)\right]\right] .
\end{aligned}
$$

Folding will happen when the second term on the righthand side of equation (13) grows sufficiently large and negative to satisfy the equality. Notice that this will happen for the first time at a position $\varphi_{\text {fold }}$ such that

$$
\varphi_{\text {fold }}=-\operatorname{Arg}(w)+\frac{\pi}{2},
$$

where

$$
w \equiv \int_{0}^{\tau} \mathrm{d} \tau_{1} A\left(\tau_{1}\right)\left(\tau-\tau_{1}\right) \exp \left[-\mathrm{i} \frac{\Omega}{\gamma}\left(\tau-\tau_{1}\right)\right] .
$$




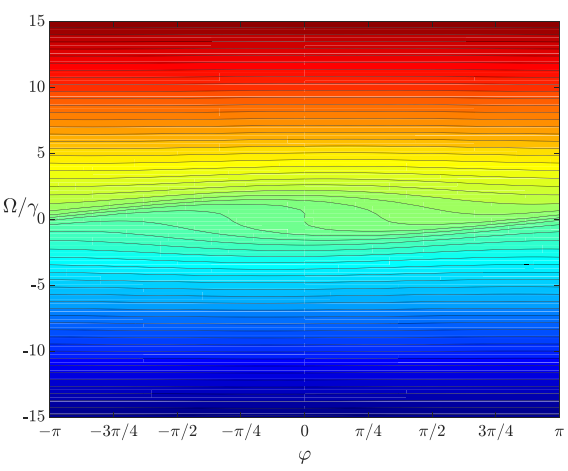

(a)

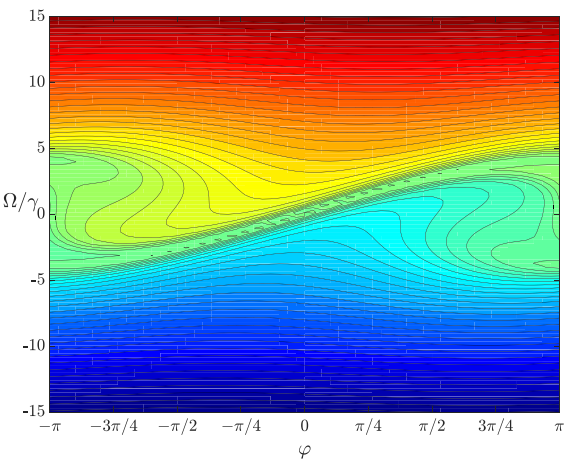

(c)

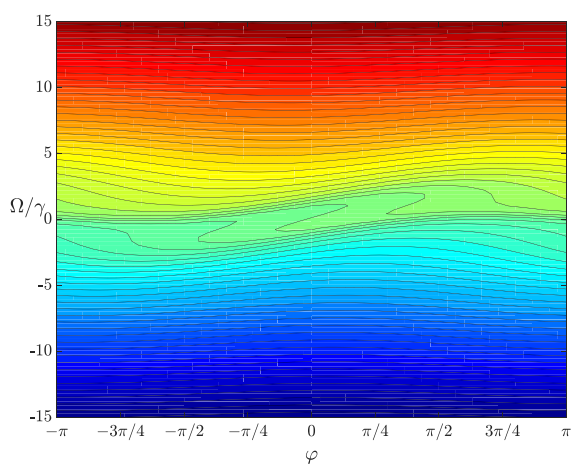

(b)

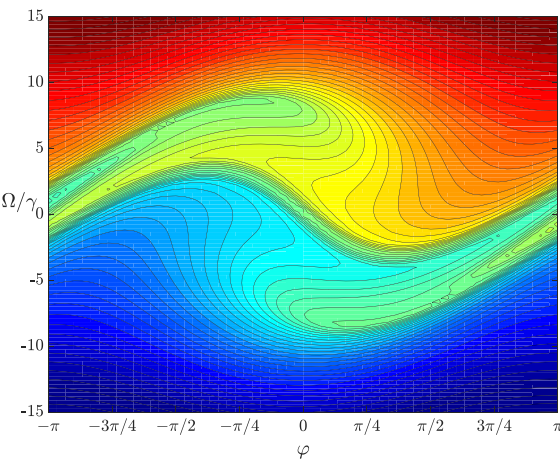

(d)

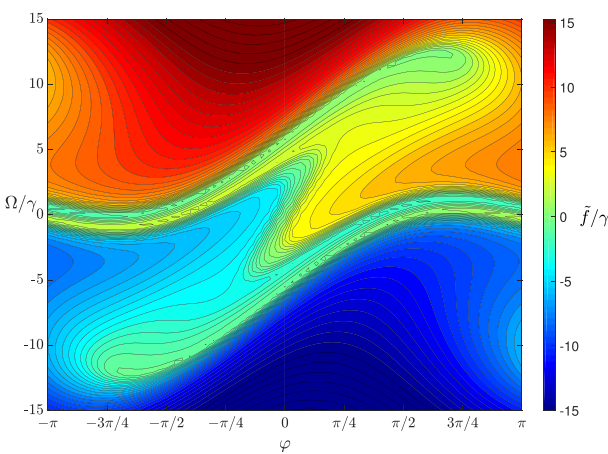

(e)

Figure 5. Distribution function contour plots at $\tau=7.41,8.17,8.84,9.29,9.57$ (red dots in figure 2) for a full-f simulation with $\gamma / \gamma_{\mathrm{L}} \approx 0.0094$. The same color scale, shown in panel $(e)$, is used throughout. Panel $(a)$ shows the moment where the distribution function folds for the first time, happening at $\varphi=0$ (O-point).

If we substitute $\varphi=\varphi_{\text {fold }}$ in equation (13) we find

$$
\frac{\gamma}{\gamma_{\mathrm{L}}}=\frac{1}{|w|^{2}} \quad \text { (at the first folding event). }
$$

We employ this expression to find the earliest time at which the distribution function for a system with ratio $\gamma / \gamma_{\mathrm{L}}$ will fold. This is done numerically in the following way. We evaluate $|w|$ over a suitable $\Omega$-domain at increasing values of time $\tau$, with $A$ being the simulated solution of the cubic equation. In this manner, we are able to identify the smallest $\tau$ for which a given value of $\gamma / \gamma_{\mathrm{L}}$ satisfies the folding condition. With $w$ being known at the time of folding, we compute the folding position $\varphi_{\text {fold }}$ through equation (14). Figure 6 shows the folding position as a function of the parameter $\gamma / \gamma_{\mathrm{L}}$. For large parameter values, the folding position is the original equilibrium position or O-point in the wave field. This result agrees with the full-Vlasov simulation that was discussed above (see the first panel of figure 5).

Immediately after the first folding event, neighboring phase space points around $\varphi_{\text {fold }}$ will begin to fold as well. Since the mode amplitude $A$ is oscillating, there will eventually be a jump in the phase of $w$ by an amount $\pm \pi$. This will trigger a separate folding event at locations $\varphi_{\text {fold }} \pm \pi$. This second event is again verified in the full-Vlasov simulation: see panel 


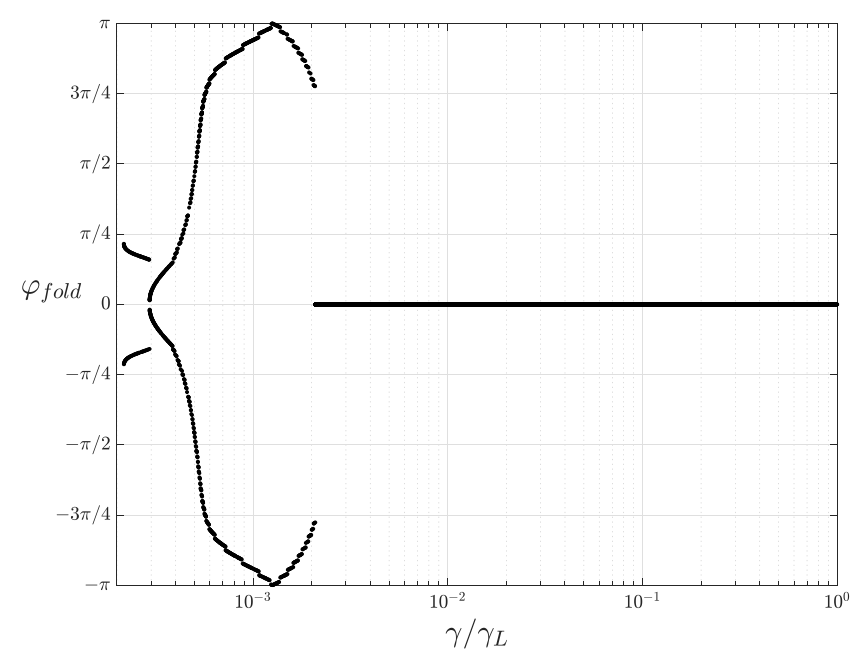

Figure 6. Predicted first folding position as a function of the small parameter $\gamma / \gamma_{\mathrm{L}}$

(c) of figure 5 at $\varphi= \pm \pi$. Notice that, for this value of $\gamma / \gamma_{\mathrm{L}}$, the cubic equation remains accurate even at the time of the second folding, as evidenced by figure 2 (this event occurs at the instant marked by the red dot at $\tau=8.84$ ).

\section{The quintic wave equation}

In this section, we will calculate the next order terms for the near-threshold wave equation (equation (1)). These new terms are quintic in the mode amplitude, and the cubic equation loses accuracy when the quintic terms compete with the cubic term. Thus, the quintic wave equation allows us to contruct a 'breakdown' criterion for the cubic equation. As we shall see, the mode amplitude level at breakdown is linked to the folding level that was discussed in the previous section.

As is well known, the Fourier component $f_{2}$ does not contribute to the cubic wave equation. Presumably, this fact is part of the robustness of the cubic equation, and it is worthwhile to understand if this is a characteristic of a deeper property. In what follows, we derive a necessary condition that tells us whether a Fourier component contributes to arbitrary order in the wave equation. This result will facilitate the calculation of the fifth-order terms.

As we have previously noted, the $n$th Fourier component is only directly coupled to its neighboring components $f_{n \pm 1}$. Since it is $f_{1}$ which enters into the wave equation, the other Fourier components will enter indirectly through a sequence of couplings. Every additional term in this sequence introduces an integration in time and increases the order in the mode amplitude by one. For example, the sequence that involves $f_{2}$ to cubic order can be schematically written as

$$
f_{1}^{(1)} \rightarrow f_{2}^{(2)} \rightarrow f_{1}^{(3)} .
$$

In this sequence, the linear $f_{1}^{(1)}$ generates the quadratic $f_{2}^{(2)}$. This in turn generates $f_{1}^{(3)}$, which is then integrated in velocity $\Omega$ to compute the current. The equilibrium gradient $F^{\prime}$ is independent of $\Omega$, and therefore, the $\Omega$-dependence of any of these Fourier components is entirely given by an exponential factor $\exp \left[\mathrm{i} \Omega h\left(t, t_{1}, t_{2}, \ldots\right)\right]$, where $h\left(t, t_{1}, t_{2}, \ldots\right)$ is a linear superposition of $t, t_{1}, t_{2}$, etc, with $t_{1,2,3, \ldots}$ are integration variables (e.g. see appendix A). Thus, the integration in $\Omega$ will result in a Dirac delta function $\delta\left(h\left(t, t_{1}, t_{2}, \ldots\right)\right)$. The argument of this delta function determines if the term contributes to the wave equation.

The exact form of $h\left(t, t_{1}, t_{2}, \ldots\right)$ can be inferred by inspecting the structure of equation (6). In the case of the term $f_{1}^{(3)}$ that results from the sequence in equation (17), the $\Omega$-dependence is given by the factor

$$
\begin{aligned}
& \exp \left[-\mathrm{i} \Omega\left(t-t_{1}\right)\right] \exp \left[-2 \mathrm{i} \Omega\left(t_{1}-t_{2}\right)\right] \exp \left[-\mathrm{i} \Omega\left(t_{2}-t_{3}\right)\right] \\
& \quad=\exp \left[-\mathrm{i} \Omega\left(t+t_{1}-t_{2}-t_{3}\right)\right]
\end{aligned}
$$

After integration over $\Omega$, the delta function obtained is thus $\delta\left(t+t_{1}-t_{2}-t_{3}\right)$. The argument of this delta function only vanishes at the upper edge of the integration domain (i.e. where all integration variables are equal to $t$ ). However, the integrand vanishes at this location of the integration domain (the integrand is shown explicitly in appendix A). This is the reason for this term not contributing to the cubic wave equation.

Now consider the case of a general $k$ th order sequence, which we write as

$f_{n_{1}}^{(1)} \rightarrow f_{n_{2}}^{(2)} \rightarrow f_{n_{3}}^{(3)} \rightarrow \ldots \rightarrow f_{n_{k-2}}^{(k-2)} \rightarrow f_{n_{k-1}}^{(k-1)} \rightarrow f_{n_{k}}^{(k)}$,

where $n_{1, \ldots, k}$ represent the sequence of harmonic numbers that generate the $k$ th order term. The $\Omega$-dependence of this general term is given by the factor

$$
\begin{gathered}
\exp \left\{-\mathrm{i} \Omega\left[n_{k}\left(t-t_{1}\right)+n_{k-1}\left(t_{1}-t_{2}\right)+n_{k-2}\left(t_{2}-t_{3}\right)\right.\right. \\
\left.\left.+\ldots+n_{2}\left(t_{k-2}-t_{k-1}\right)+n_{1}\left(t_{k-1}-t_{k}\right)\right]\right\}
\end{gathered}
$$

After integration over $\Omega$ is done, the delta function that is generated is

$$
\begin{array}{r}
\delta\left(n_{k}\left(t-t_{1}\right)+n_{k-1}\left(t_{1}-t_{2}\right)+n_{k-2}\left(t_{2}-t_{3}\right)\right. \\
\left.+\ldots+n_{2}\left(t_{k-2}-t_{k-1}\right)+n_{1}\left(t_{k-1}-t_{k}\right)\right) .
\end{array}
$$

Notice that if $n_{1, \ldots, k}$ are all either positive or negative, then the argument of the delta function is everywhere positive or negative, except at the edge of the integration domain, where all integration variables are equal to $t$. Now, if we look at equation (6), we see that $f_{n}$ depends upon $f_{n+1}^{\prime}$. Making the substitution $n \rightarrow n \pm 1$ in that same equation and taking its derivative with respect to $\Omega$, we find that there is a factor of $\left(t-t_{1}\right)$ in the integrand of $f_{n \pm 1}^{\prime}$ and thus in that of $f_{n}$. Therefore, except for $f_{1}^{(1)}$ (equation (8)), the integrand associated with any Fourier component will always vanish when all integration variables are equal to $t$.

Thus we infer that if $n_{1, \ldots, k}$ are all either positive or negative, then the sequence in question will not contribute to the wave equation. We can then state the following corollary: for a sequence to contribute to the wave equation to any order higher than one, it must contain $f_{0}$. This is true because we need to have at least one negative and one positive Fourier harmonic (i.e. at least one component $f_{n}$ with $n<0$, and at least one with $n>0$ ). Since every Fourier component $f_{n}$ only directly couples to its neighboring components $f_{n \pm 1}$, we must 
have $f_{0}$ present to be able to include both negative and positive harmonics in the sequence. QED.

The observation in the previous paragraph explains, in a general way, why $f_{2}$ does not enter into the cubic equation (indeed, note that $f_{0}$ is not present in equation (17)). We can also use this observation to write down the sequences of couplings that will contribute to the fifth order terms in the wave equation. These sequences are

$$
\begin{aligned}
& f_{1}^{(1)} \rightarrow f_{0}^{(2)} \rightarrow f_{-1}^{(3)} \rightarrow f_{0}^{(4)} \rightarrow f_{1}^{(5)}, \\
& f_{-1}^{(1)} \rightarrow f_{0}^{(2)} \rightarrow f_{1}^{(3)} \rightarrow f_{0}^{(4)} \rightarrow f_{1}^{(5)}, \\
& f_{-1}^{(1)} \rightarrow f_{0}^{(2)} \rightarrow f_{-1}^{(3)} \rightarrow f_{0}^{(4)} \rightarrow f_{1}^{(5)} . \\
& f_{-1}^{(1)} \rightarrow f_{0}^{(2)} \rightarrow f_{1}^{(3)} \rightarrow f_{2}^{(4)} \rightarrow f_{1}^{(5)}, \\
& f_{-1}^{(1)} \rightarrow f_{-2}^{(2)} \rightarrow f_{-1}^{(3)} \rightarrow f_{0}^{(4)} \rightarrow f_{1}^{(5)} .
\end{aligned}
$$

Notice that the components $f_{n}$ with $n \geqslant 3$ do not enter into the wave equation even to fifth order. Whereas to cubic order there is only one sequence that contributes to the wave equation (namely $f_{-1}^{(1)} \rightarrow f_{0}^{(2)} \rightarrow f_{1}^{(3)}$ ), to fifth order there are five sequences. Furthermore, the quintic terms involve quadruple integrations over past history. Thus, these terms are of considerable complexity when compared to the cubic one. The fifth order wave equation is

$$
\begin{aligned}
\frac{\mathrm{d} A}{\mathrm{~d} \tau}= & A(\tau)-\frac{1}{2} \int_{0}^{\tau / 2} \mathrm{~d} \tau_{1} \tau_{1}^{2} A\left(\tau-\tau_{1}\right) \\
& \times \int_{0}^{\tau-2 \tau_{1}} \mathrm{~d} \tau_{2} A\left(\tau-\tau_{1}-\tau_{2}\right) A^{*}\left(\tau-2 \tau_{1}-\tau_{2}\right) \\
& +\frac{1}{8} \frac{\gamma}{\gamma_{\mathrm{L}}}\left(Q_{1}+Q_{2}+Q_{3}+Q_{4}+Q_{5}\right),
\end{aligned}
$$

where each of the five quintic terms of the form $\left(\gamma /\left(8 \gamma_{\mathrm{L}}\right)\right) Q_{i}$ corresponds to a sequence in equation (22). The $Q_{i}$ terms only depend upon $A$ and $\tau$, and are given explicitly in appendix B.

A straight-forward simulation of equation (23) can be computationally expensive. However, one can use the simulated solution of the cubic equation to compute the quintic terms $Q_{i}$ once and for all, and then treat these terms as sources in equation (23). We have solved the quintic both in this perturbative manner and in a straight-forward fashion, and have found that the difference is indeed negligible. This is to be expected if we are working within the near-threshold regime.

In figure 7 we show, in dashed green, the simulated output of simulations of equation (23) for two different values of $\gamma / \gamma_{\mathrm{L}}$. On the same figure, also shown are the cubic solution (blue) and the full-Vlasov simulation output (red), the latter having the same $\gamma / \gamma_{\mathrm{L}}$ value as the one used in the quintic equation. The three solutions have been shifted in time to match them at the first maximum, at which point the evolution is nonlinear; figure 7 only shows this nonlinear part of the evolution. Notice that the quintic solution follows the full-Vlasov

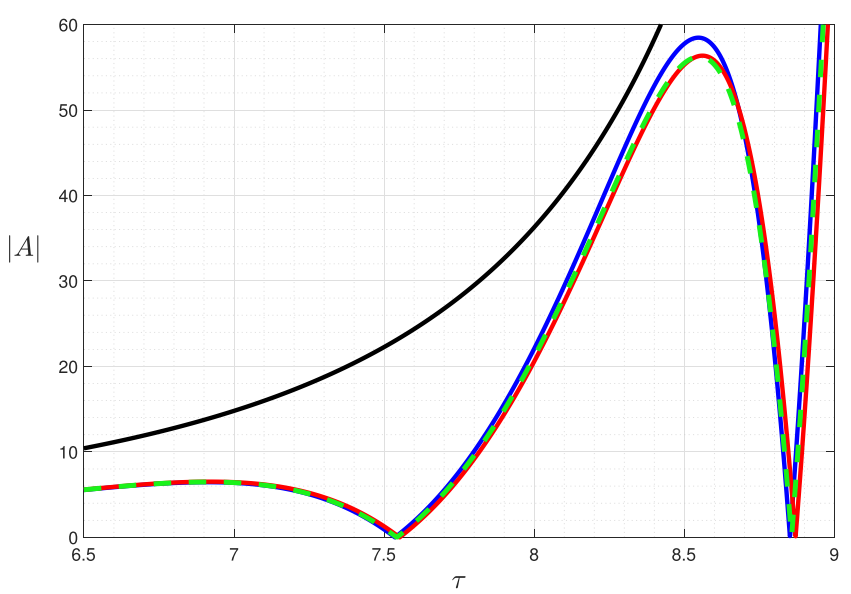

(a)

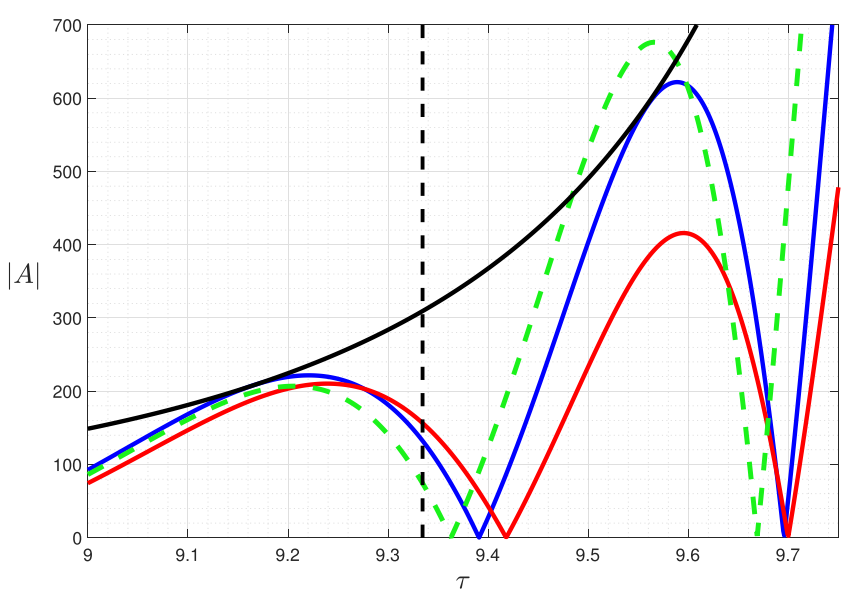

(b)

Figure 7. Evolution of the mode amplitude as given by fullVlasov simulation (red), quintic equation (dashed green), and cubic equation (blue), with $\gamma / \gamma_{\mathrm{L}} \approx 0.0094$. Also shown, in solid black, is the envelope of the cubic solution. (a) Early nonlinear evolution, where the quintic solution is seen to replicate the full-Vlasov simulation more closely. (b) Later stages of the same simulation (note the change in vertical scale). The vertical line marks the point where the relative error between cubic and quintic solutions reaches $18 \%$ (see equation (25)).

simulation more closely than does the cubic solution at the beginning of the nonlinear stage, as is to be expected. The vertical line in figure 7 indicates that the relative error between cubic and quintic solutions has reached $18 \%$, a working criterion that we use to determine the breakdown of the cubic equation. We will expound on this point shortly. On the other hand, the saturation level of the full-Vlasov simulation was shown to be $\omega_{b \text {;sat }}=16 \gamma_{\mathrm{L}} /\left(3 \pi^{2}\right)$ in [14] (and this level is verified in our simulations). In terms of $A$, this level is

$$
A_{\mathrm{sat}}=\omega_{b ; \mathrm{sat}}^{2} \frac{\gamma_{\mathrm{L}}^{1 / 2}}{\gamma^{5 / 2}}=\frac{256}{9 \pi^{4}}\left(\frac{\gamma_{\mathrm{L}}}{\gamma}\right)^{5 / 2} .
$$




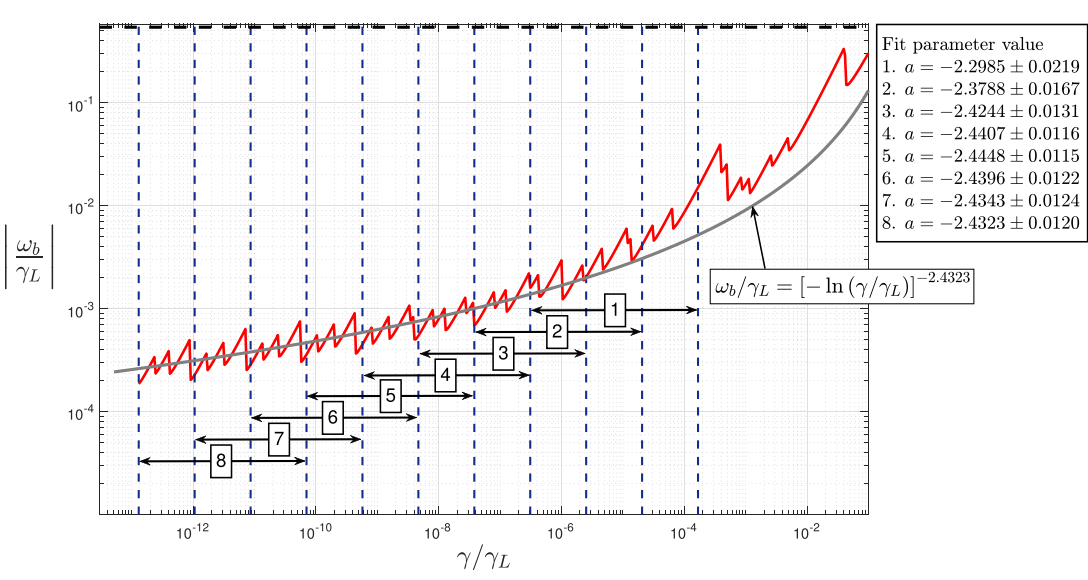

Figure 8. The red curve represents the amplitude level at which the deviation error between cubic and quintic solutions (equation (25)) reaches $18 \%$. This curve is fitted with a function of the form $\left|\omega_{b} / \gamma_{L}\right|=\left[-\ln \left(\gamma / \gamma_{L}\right)\right]^{a}$ in each of the eight intervals shown. The fit parameter values in each of these intervals is shown on the right, together with $95 \%$ confidence bounds. The smooth curve represents the best fit solution that is found at the eighth interval. The saturation level is shown as a horizontal dashed line.

For the case shown in figure 7 , the saturation amplitude is approximately $A_{\text {sat }} \approx 3.44 \times 10^{4}$, which we can see is well beyond the scales shown in the figure. The cubic equation was previously assumed to hold its accuracy up to the saturation level, but here we see that it loses accuracy prematurely.

The simulated solution to the quintic equation is initially indistinguishable from that of the cubic equation. As the mode amplitude level increases, the quintic terms grow, and at some point, their size relative to the cubic term, though still small, becomes appreciable. When this happens, the quintic terms contribute a significant correction to the mode amplitude, and the response follows the full-Vlasov simulation more closely than does the cubic solution. Eventually, the cubic and quintic terms reach the same level, breaking the assumed hierarchy of the nonlinear terms in the wave equation. At this level, the cubic equation loses its accuracy. After this happens, the simulated quintic solution eventually becomes larger than the cubic solution (see figure 7). This observation is explained by the fact that an explosive solution $|A| \sim\left(\tau_{0}-\tau\right)^{-5 / 2}$ has been excited with an earlier blow-up time $\tau_{0}$ than the one of the cubic solution; this is an intrinsic instability of the explosive solutions [9]. In this regime, the cubic term again dominates the quintic term, and the amplitude level is beyond the scope of the theory.

From the previous discussion, we see that an adequate criterion by which to measure breakdown is to look for a significant deviation between cubic and quintic solutions. We define the relative error $\epsilon$ between the cubic solution, $A_{\mathrm{c}}$, and the quintic solution, $A_{\mathrm{q}}$, as

$$
\epsilon=\frac{\left|A_{\mathrm{q}}-A_{\mathrm{c}}\right|}{\text { Env }}
$$

where Env $\propto\left(\tau_{0}-\tau\right)^{-5 / 2}$ is the envelope of the cubic solution, which is an adequate measure of the size of the mode amplitude (this envelope is shown in figure 7). We establish, as

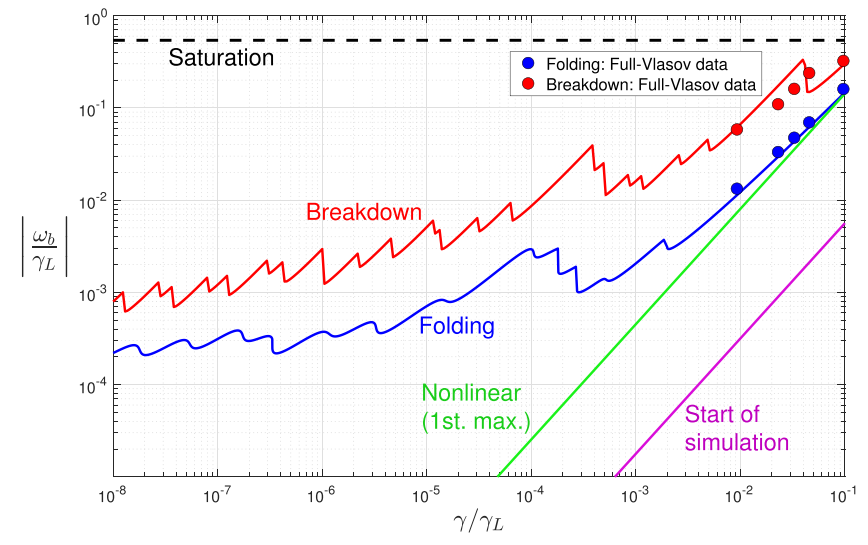

Figure 9. The curves represent the amplitude levels at: the start of the simulations, the onset of the nonlinear behavior, the first folding event, the breakdown of the cubic equation, and saturation. Dots represent data from full-Vlasov simulations.

a working criterion, that the cubic equation has broken down when an $18 \%$ error has been reached, i.e. when $\epsilon=0.18$. We have chosen this value for the criterion based on what we consider to be a 'sufficiently-large' deviation; the value is not important for the conclusions that we derive.

We use this criterion to find the variation of the breakdown level as a function of $\gamma / \gamma_{\mathrm{L}}$, and the results are represented by a red curve in figure 8 . As a reference, the horizontal dotted line indicates the saturation level of the full-Vlasov simulation, which as previously stated, is $\omega_{b \text {;sat }}=16 \gamma_{\mathrm{L}} /\left(3 \pi^{2}\right)$ [14]. It is worth noting at this point that computer simulations with uniformly-spaced grids in time are inadequate because the magnitude and the frequency of the mode amplitude increase indefinitely. The appropriate variable that resolves the mode amplitude as we get arbitrarily close to blow-up is $\ln \left(\tau_{0}-\tau\right)$ [9]. This is especially important when very small values of $\gamma / \gamma_{\mathrm{L}}$ are used, because breakdown happens increasingly closer to the blow-up time. 
Note that for the smallest $\gamma / \gamma_{\mathrm{L}}$ values shown in figure 8 , a steady behavior appears to emerge (roughly for $\left.\gamma / \gamma_{\mathrm{L}} \lesssim 10^{-9}\right)$. We find that as $\gamma / \gamma_{\mathrm{L}}$ decreases, the breakdown level approaches a logarithmic scaling of the form $\left|\omega_{b} / \gamma_{\mathrm{L}}\right|=\left[-\ln \left(\gamma / \gamma_{\mathrm{L}}\right)\right]^{a}$, where $a$ is a fit parameter that we determine through a least-squares routine. To test this scaling, we divide the $\gamma / \gamma_{\mathrm{L}}$ domain into the eight intervals shown in figure 8 , and we find the fit parameter $a$ in each of these segments. The values are shown to the right of the figure, together with uncertainties which define $95 \%$ confidence bounds. The best-fit curve corresponding to the eighth interval (where $a=-2.4323 \pm 0.0120)$ is plotted on the same figure.

Figure 9 displays the breakdown level together with the amplitude level where folding is first predicted to happen (in blue) according to the equations of section 2 . Recall that this is a predictive scheme where we use the linear part of the perturbed distribution function to find the first instance where $\partial f / \partial \Omega=0$. For sufficiently small $\gamma / \gamma_{\mathrm{L}}$ values, this method is not adequate because the nonlinear Fourier components of $f$ begin to compete with the linear part. It is found that when $\gamma / \gamma_{\mathrm{L}} \lesssim 10^{-8}$, the linear and nonlinear components become of order the equilibrium distribution at approximately the same time. Therefore, we only show larger $\gamma / \gamma_{\mathrm{L}}$ values in figure 9, where the predictive scheme is trusted. We see that folding happens before the cubic equation loses its accuracy.

Figure 9 also shows the amplitude level corresponding to the first local maximum of $|A|$. This level is taken as a simple indication of the fact that the evolution has entered its nonlinear stage. Computer simulations of the cubic equation indicate that the first peak value converges to $\left|A_{\text {peak }}\right| \approx 6.46$ for decreasing simulation step-size. Thus, in view of the relation $A=\omega_{b}^{2} \gamma_{\mathrm{L}}^{1 / 2} / \gamma^{5 / 2}$, we obtain $\left|\omega_{b, \text { peak }} / \gamma_{\mathrm{L}}\right| \approx 2.54\left(\gamma / \gamma_{\mathrm{L}}\right)^{5 / 4}$, which is represented by the green line in figure 9 . Our simulations are always initiated in the linear stage, at the level represented by the purple line in the same figure. Note that while the breakdown level decreases with smaller values of $\gamma / \gamma_{\mathrm{L}}$, the window of nonlinear behavior that is captured by the cubic equation (i.e. the separation between nonlinear and breakdown curves) increases, as expected.

Finally, we also compute the breakdown level by calculating the error between the cubic and full-Vlasov solutions. We make use of equation (25) replacing the quintic solution $A_{\mathrm{q}}$ with the full-Vlasov solution, and use the same working criterion as before (18\%). We show the breakdown level for five full-Vlasov simulations through the red dots in figure 9. Note that the dots follow the behavior that was predicted through a comparison between cubic and quintic solutions. For the same full-Vlasov runs, we find the first instance of folding and plot the corresponding amplitude levels as blue dots. Note that in the full-Vlasov simulations, folding arises at an amplitude level that is very close as that predicted by the perturbative scheme as given by the solid blue curve. These observations allow us to verify the appropriateness of our breakdown and folding criteria.

\section{Conclusion}

In this work, we have studied the breakdown of the nearthreshold theory for kinetic instabilities first derived in [1]. We have shown that the mode amplitude predicted by the cubic equation of said theory loses accuracy below the expected saturation level $\left|\omega_{b}\right| \sim \gamma_{\mathrm{L}}$ of the full-Vlasov solution. The loss in accuracy has been quantified by computing the deviation between predictions of the cubic and the newlyderived quintic equations. A sufficiently large deviation is considered a breakdown of the theory because the hierarchy of the terms in the wave equation is broken. In this manner, we are able to analyze the loss of accuracy arbitrarily close to marginal stability (as measured by $\gamma / \gamma_{\mathrm{L}}$ ), something that is computationally unfeasible with full-Vlasov simulations. For sufficiently large $\gamma / \gamma_{\mathrm{L}}$, however, we are able to compute the deviation between cubic equation and full-Vlasov simulations, and we find good agreement with the breakdown level predicted through comparisons of the cubic and quintic equations.

We have found that a folding in the contour levels of the distribution function (i.e. $\partial f / \partial \Omega=0$ ) occurs before the weaklynonlinear theory breaks down. The amplitude level at which folding occurs varies with closeness to marginal stability, and we have studied this level through both a perturbative scheme and full nonlinear simulations, finding good agreement between the two procedures. The advantage of the perturbative scheme is that we are able to study arbitrarily small values of $\gamma / \gamma_{\mathrm{L}}$. We find that folding always occurs before the quintic terms in the wave equation become important (i.e. while the cubic equation is a good approximation).

Thus we have found two unexpected results: that the weakly-nonlinear theory loses accuracy prematurely, and that folding happens well within the validity of this theory. An additional, related result is suggested by our findings, as there appears to be a causal connection between the first two results. That is, folding appears to cause the theory's loss of accuracy. The breakdown of the theory's quantitative accuracy arises when the bounce frequency $\left|\omega_{b}\right|$ is a factor of 5-10 above the level where the first fold appears (see figure 9), for sufficiently small $\gamma / \gamma_{\mathrm{L}}$. The details of this link still need to be uncovered, but we offer a conjecture. As explained in [9], the explosive solutions of the cubic wave equation have an intrinsic 'symmetry' instability, where solutions with slightly different blow-up times deviate in time as $1 /\left(t_{0}-t\right)$. Thus one possible explanation for the premature accuracy loss of the explosive solutions is the onset of such an instability. If that is the case, then the folding phenomenon described in the present work can be identified as a trigger to the instability.

\section{Appendix A. Fourier components of the perturbed distribution function}

Up to cubic order in the mode amplitude, 
AQ9

$$
\begin{aligned}
& f_{1}^{(1)}=-\frac{F^{\prime}}{2} \int_{0}^{t} \mathrm{~d} t_{1} \omega_{b}^{2}\left(t_{1}\right) \exp \left[-\mathrm{i} \Omega\left(t-t_{1}\right)\right] \\
& f_{0}^{(2)}=-\frac{F^{\prime}}{2} \int_{0}^{t} \mathrm{~d} t_{1} \omega_{b}^{2}\left(t_{1}\right) f_{1}^{\prime *(1)}+\text { c.c. } \\
& =\frac{\mathrm{i} F^{\prime}}{4} \int_{0}^{t} \mathrm{~d} t_{1} \omega_{b}^{2}\left(t_{1}\right) \int_{0}^{t_{1}} \mathrm{~d} t_{2} \omega_{b}^{2 *}\left(t_{2}\right)\left(t_{1}-t_{2}\right) \exp \left[\mathrm{i} \Omega\left(t_{1}-t_{2}\right)\right]+\text { c.c. } \\
& f_{2}^{(2)}=-\frac{F^{\prime}}{2} \int_{0}^{t} \mathrm{~d} t_{1} \omega_{b}^{2}\left(t_{1}\right) f_{1}^{\prime(1)} \exp \left[-2 \mathrm{i} \Omega\left(t-t_{1}\right)\right] \\
& =-\frac{\mathrm{i} F^{\prime}}{4} \int_{0}^{t} \mathrm{~d} t_{1} \omega_{b}^{2}\left(t_{1}\right) \int_{0}^{t_{1}} \mathrm{~d} t_{2} \omega_{b}^{2}\left(t_{2}\right)\left(t_{1}-t_{2}\right) \exp \left[-\mathrm{i} \Omega\left(2 t-t_{1}-t_{2}\right)\right] \\
& f_{1}^{(3)}=-\frac{F^{\prime}}{2}\left\{\int_{0}^{t} \mathrm{~d} t_{1} \omega_{b}^{2}\left(t_{1}\right) f_{0}^{\prime(2)} \exp \left[-\mathrm{i} \Omega\left(t-t_{1}\right)\right]+\int_{0}^{t} \mathrm{~d} t_{1} \omega_{b}^{2 *}\left(t_{1}\right) f_{2}^{\prime(2)} \exp \left[-\mathrm{i} \Omega\left(t-t_{1}\right)\right]\right\} \\
& =\frac{F^{\prime}}{8} \int_{0}^{t} \mathrm{~d} t_{1} \omega_{b}^{2}\left(t_{1}\right) \exp \left[-\mathrm{i} \Omega\left(t-t_{1}\right)\right] \\
& \times\left\{\int_{0}^{t_{1}} \mathrm{~d} t_{2} \omega_{b}^{2}\left(t_{2}\right) \int_{0}^{t_{2}} \mathrm{~d} t_{3} \omega_{b}^{2 *}\left(t_{3}\right)\left(t_{2}-t_{3}\right)^{2} \exp \left[\mathrm{i} \Omega\left(t_{2}-t_{3}\right)\right]+\text { c.c. }\right\} \\
& +\frac{F^{\prime}}{8} \int_{0}^{t} \mathrm{~d} t_{1} \omega_{b}^{2 *}\left(t_{1}\right) \int_{0}^{t_{1}} \mathrm{~d} t_{2} \omega_{b}^{2}\left(t_{2}\right) \\
& \times \int_{0}^{t_{2}} \mathrm{~d} t_{3} \omega_{b}^{2}\left(t_{3}\right)\left[\left(2 t_{1}-t_{2}-t_{3}\right)\left(t_{2}-t_{3}\right)\right] \exp \left[-\mathrm{i} \Omega\left(t+t_{1}-t_{2}-t_{3}\right)\right] \\
& f_{3}^{(3)}=-\frac{F^{\prime}}{2} \int_{0}^{t} \mathrm{~d} t_{1} \omega_{b}^{2}\left(t_{1}\right) f_{2}^{\prime(2)} \exp \left[-3 \mathrm{i} \Omega\left(t-t_{1}\right)\right] \\
& =\frac{F^{\prime}}{8} \int_{0}^{t} \mathrm{~d} t_{1} \omega_{b}^{2}\left(t_{1}\right) \int_{0}^{t_{1}} \mathrm{~d} t_{2} \omega_{b}^{2}\left(t_{2}\right) \\
& \times \int_{0}^{t_{2}} \mathrm{~d} t_{3} \omega_{b}^{2}\left(t_{3}\right)\left[\left(2 t_{1}-t_{2}-t_{3}\right)\left(t_{2}-t_{3}\right)\right] \exp \left[-\mathrm{i} \Omega\left(3 t-t_{1}-t_{2}-t_{3}\right)\right] .
\end{aligned}
$$

\section{Appendix B. Fifth order terms in the wave equation}

The fifth-order terms in the wave equation, equation (23), are

$$
\begin{aligned}
& Q_{1}=\int_{\tau / 2}^{\tau} \mathrm{d} \tau_{1} A\left(\tau_{1}\right) \int_{\tau-\tau_{1}}^{\tau_{1}} \mathrm{~d} \tau_{2} A\left(\tau_{2}\right) \int_{-\tau+\tau_{1}+\tau_{2}}^{\tau_{2}} \mathrm{~d} \tau_{3} A^{*}\left(\tau_{3}\right) \int_{\tau-\tau_{1}-\tau_{2}+\tau_{3}}^{\tau_{3}} \mathrm{~d} \tau_{4} K_{1} A\left(\tau_{4}\right) A^{*}\left(-\tau+\tau_{1}+\tau_{2}-\tau_{3}+\tau_{4}\right), \\
& Q_{2}=\int_{\tau / 2}^{\tau} \mathrm{d} \tau_{1} A\left(\tau_{1}\right) \int_{\tau-\tau_{1}}^{\tau_{1}} \mathrm{~d} \tau_{2} A\left(\tau_{2}\right) \int_{\left(-\tau+\tau_{1}+\tau_{2}\right) / 2}^{-\tau+\tau_{1}+\tau_{2}} \mathrm{~d} \tau_{3} A^{*}\left(\tau_{3}\right) \int_{-\tau+\tau_{1}+\tau_{2}-\tau_{3}}^{\tau_{3}} \mathrm{~d} \tau_{4} K_{1} A^{*}\left(\tau_{4}\right) A\left(\tau-\tau_{1}-\tau_{2}+\tau_{3}+\tau_{4}\right) \text {, } \\
& Q_{3}=\int_{\tau / 2}^{\tau} \mathrm{d} \tau_{1} A\left(\tau_{1}\right) \int_{\tau-\tau_{1}}^{\tau_{1}} \mathrm{~d} \tau_{2} A^{*}\left(\tau_{2}\right) \int_{\left(\tau-\tau_{1}+\tau_{2}\right) / 2}^{\tau_{2}} \mathrm{~d} \tau_{3} A\left(\tau_{3}\right) \quad \int_{\tau-\tau_{1}+\tau_{2}-\tau_{3}}^{\tau_{3}} \mathrm{~d} \tau_{4} K_{2} A\left(\tau_{4}\right) A^{*}\left(-\tau+\tau_{1}-\tau_{2}+\tau_{3}+\tau_{4}\right), \\
& Q_{4}=\int_{\tau / 2}^{\tau} \mathrm{d} \tau_{1} A^{*}\left(\tau_{1}\right) \int_{\left(\tau+\tau_{1}\right) / 3}^{\tau_{1}} \mathrm{~d} \tau_{2} A\left(\tau_{2}\right) \int_{\left(\tau+\tau_{1}-\tau_{2}\right) / 2}^{\tau_{2}} \mathrm{~d} \tau_{3} A\left(\tau_{3}\right) \int_{\tau+\tau_{1}-\tau_{2}-\tau_{3}}^{\tau_{3}} \mathrm{~d} \tau_{4} K_{3} A\left(\tau_{4}\right) A^{*}\left(-\tau-\tau_{1}+\tau_{2}+\tau_{3}+\tau_{4}\right), \\
& Q_{5}=\int_{\tau / 3}^{\tau / 2} \mathrm{~d} \tau_{1} A^{*}\left(\tau_{1}\right) \int_{\left(\tau-\tau_{1}\right) / 2}^{\tau_{1}} \mathrm{~d} \tau_{2} A^{*}\left(\tau_{2}\right) \int_{\tau-\tau_{1}-\tau_{2}}^{\tau_{2}} \mathrm{~d} \tau_{3} A\left(\tau_{3}\right) \quad \int_{\left(-\tau+\tau_{1}+\tau_{2}+\tau_{3}\right) / 2}^{-\tau+\tau_{1}+\tau_{2}+\tau_{3}} \mathrm{~d} \tau_{4} K_{4} A\left(\tau_{4}\right) A\left(-\tau+\tau_{1}+\tau_{2}+\tau_{3}-\tau_{4}\right) \\
& +\int_{\tau / 2}^{\tau} \mathrm{d} \tau_{1} A^{*}\left(\tau_{1}\right) \int_{\left(\tau-\tau_{1}\right) / 2}^{\tau-\tau_{1}} \mathrm{~d} \tau_{2} A^{*}\left(\tau_{2}\right) \int_{\tau-\tau_{1}-\tau_{2}}^{\tau_{2}} \mathrm{~d} \tau_{3} A\left(\tau_{3}\right) \quad \int_{\left(-\tau+\tau_{1}+\tau_{2}+\tau_{3}\right) / 2}^{-\tau+\tau_{1}+\tau_{2}+\tau_{3}} \mathrm{~d} \tau_{4} K_{4} A\left(\tau_{4}\right) A\left(-\tau+\tau_{1}+\tau_{2}+\tau_{3}-\tau_{4}\right) \\
& +\int_{\tau / 2}^{\tau} \mathrm{d} \tau_{1} A^{*}\left(\tau_{1}\right) \int_{\tau-\tau_{1}}^{\tau_{1}} \mathrm{~d} \tau_{2} A^{*}\left(\tau_{2}\right) \int_{-\tau+\tau_{1}+\tau_{2}}^{\tau_{2}} \mathrm{~d} \tau_{3} A\left(\tau_{3}\right) \quad \int_{\left(-\tau+\tau_{1}+\tau_{2}+\tau_{3}\right) / 2}^{\tau_{3}} \mathrm{~d} \tau_{4} K_{4} A\left(\tau_{4}\right) A\left(-\tau+\tau_{1}+\tau_{2}+\tau_{3}-\tau_{4}\right)
\end{aligned}
$$

where the kernels are 


$$
\begin{aligned}
& K_{1}=\left(\tau-\tau_{1}\right)^{2}\left(\tau-\tau_{1}-\tau_{2}+\tau_{3}\right)^{2}, \\
& K_{2}=\left(\tau-\tau_{1}\right)^{2}\left(\tau-\tau_{1}+\tau_{2}-\tau_{3}\right)^{2} \\
& K_{3}=\left(\tau-\tau_{1}\right)\left(\tau+\tau_{1}-2 \tau_{2}\right)\left(\tau+\tau_{1}-\tau_{2}-\tau_{3}\right)^{2}, \\
& K_{4}=\left(\tau-\tau_{1}\right)^{2}\left(\tau-\tau_{1}-\tau_{2}+\tau_{3}\right)\left(\tau-\tau_{1}-\tau_{2}-\tau_{3}+2 \tau_{4}\right) .
\end{aligned}
$$

\section{ORCID iDs}

D. Sanz-Orozco 누 https://orcid.org/0000-0002-1430-6073

\section{References}

[1] Berk H.L., Breizman B.N. and Pekker M. 1996 Nonlinear dynamics of a driven mode near marginal stability Phys. Rev. Lett. 76 1256-9

[2] Breizman B.N., Berk H.L., Pekker M.S., Porcelli F., Stupakov G.V. and Wong K.L. 1997 Critical nonlinear phenomena for kinetic instabilities near threshold Phys. Plasmas 4 1559-68

[3] Berk H.L., Breizman B.N. and Pekker M.S. 1997 Nonlinear theory of kinetic instabilities near threshold Plasma Phys. Rep. 23 778-88

[4] Idouakass M. 2016 Linear and nonlinear study of the precessional fishbone instability $P h D$ Thesis Aix-Marseille University

[5] Wong K.L., Majeski R., Petrov M., Rogers J.H., Schilling G., Wilson J.R., Berk H.L., Breizman B.N., Pekker M. and Wong H.V. 1997 Evolution of toroidal alfvén eigenmode instability in tokamak fusion test reactor Phys. Plasmas 4 393-404

[6] Fasoli A., Breizman B.N., Borba D., Heeter R.F., Pekker M.S and Sharapov S.E. 1998 Nonlinear splitting of fast particle driven waves in a plasma: observation and theory Phys. Rev. Lett. 81 5564-7

[7] Heeter R.F., Fasoli A.F. and Sharapov S.E. 2000 Chaotic regime of alfvén eigenmode wave-particle interaction Phys. Rev. Lett. 85 3177-80

[8] Heeter R.F. 1999 PhD Thesis Princeton University

[9] Sanz-Orozco D. and Berk H.L. 2017 Explosive attractor solutions to a universal cubic delay equation Phys. Plasmas 24055701

[10] Lilley M.K., Breizman B.N. and Sharapov S.E. 2009 Destabilizing effect of dynamical friction on fast-particledriven waves in a near-threshold nonlinear regime Phys. Rev. Lett. 102195003

[11] Lilley M.K., Breizman B.N. and Sharapov S.E. 2010 Effect of dynamical friction on nonlinear energetic particle modes Phys. Plasmas 17092305

[12] Imadera K., Kishimoto Y., Saito D., Li J. and Utsumi T. 2009 A numerical method for solving the Vlasov-Poisson equation based on the conservative IDO scheme J. Comput. Phys. 228 8919-43

[13] Press W.H., Teukolsky S.A., Vetterling W.T. and Flannery B.P. 1992 Numerical Recipes in FORTRAN. The Art of Scientific Computing

[14] Berk H., Breizman B. and Petviashvili N. 1997 Spontaneous hole-clump pair creation in weakly unstable plasmas Phys. Lett. A 234 213-8

Berk H., Breizman B. and Petviashvili N. 1998 Spontaneous hole-clump pair creation in weakly unstable plasmas Phys. Lett. A 238408 (erratum) 